\title{
Reduction of the guessing pool in paired-associate learning*
}

\author{
RICHARD S. KEISTER † and JAMES R. ERICKSON \\ Human Performance Center, Ohio State University, Columbus, Ohio 43210
}

The Ss learned two 12-item paired-associate lists under self-paced conditions where the response pool was available. Analysis focused on S's precriterion responses. Responses learned to items tended not to be made as guesses on other items, but this type of response-pool reduction was not sufficient to account for nonstationarity of the precriterion learning curve. Other sources of response-pool reduction such as partial learning of the correct response or stimulus generalization errors seem capable of accounting for the observed amount of nonstationarity.

During the precriterion period-the period before the trial of last error for a given item in a paired-associate list-the probability of a correct response does not ordinarily remain stationary at a chance level except under very simple circumstances, e.g., when there are only two possible responses to the various stimuli. Usually the probability of a correct response increases, particularly during the latter part of the precriterion period (e.g., Suppes \& Ginsberg, 1963). Among possible explanations for this fact have been suggestions that, as learning of the list progresses, Ss become more efficient at guessing the (unknown) correct responses to the remaining unlearned items.

Along these lines, O'Hara \& Erickson (1969) presented evidence that Ss can learn something about which responses are not correct for a given stimulus and eliminate these from the guessing pool [as proposed by the models of Millward (1964) and Nahinsky (1967)]. However, they also showed that error elimination was not sufficient to account for all of the nonstationarity, even in their simple 12-stimulus 3-response task.

Another suggestion regarding increased guessing efficiency was offered by Bower (1962), who proposed that, at least in a list having an equal number of stimuli and responses, the probability of a correct guess might be close to $1 / N-k$, where $N$ is the number of response alternatives and $k$ is the number of learned items. In other words, as Ss learn which alternatives are correct to given stimuli, they eliminate them from the guessing pools for other yet

*This research was carried out at the Human Performance Center, the Ohio State University, and was supported in part by National Institutes of Health Grant No. MH-12113. The Computer Centers of Ohio State University and Indiana State University provided free time for data analysis, for which we are grateful.

+Now at Indiana State University. Terre Haute. Ind. 47809 . unlearned items. There exists evidence (Bregman, 1966) that in a recognition-memory task, Ss can make use of information reducing the size of the guessing pool in this manner, but there seems to be little or no direct evidence regarding this kind of guessing-pool shrinkage in a standard paired-associate learning task. In this article we present such evidence and show the extent to which this type of increased guessing efficiency can account for improved performance during the precriterion period, i.e., for nonstationarity.

\section{METHOD}

The Ss were 20 males from the introductory psychology $\mathbf{S}$ pool at the Ohio State University. These Ss volunteer for particular experiments in fulfillment of a course requirement of experimental participation.

The stimuli for each list were 12 randomly chosen consonants, and responses were randomly chosen two-digit numbers. List $A$ consisted of the following pairs: F-12, D-18, Z-24, $\mathrm{V}-27, \mathrm{X}-45, \mathrm{Q}-47, \mathrm{~S}-52, \mathrm{H}-66, \mathrm{~N}-71$, B-73, L-81, and J-96. The pairs in List B were B-14, N-21, L-26, S-34, G-42, V-56, W-63, H-64, M-72, P-79, F-83, and Z-85.

The materials were presented on a Lafayette memory drum equipped with a remote control so that Ss had an unlimited time to respond to each stimulus term; following the response, the correct alternative was shown for a 2 sec period, after which the next stimulus term was exposed.

Each S learned two lists, Lists A and $B$ composed as above, to a criterion of two perfect trials through the list. In order to provide response availability, before learning one list, Ss studied the response alternatives until they could recall them perfectly, and before learning the other list, a card giving the possible response alternatives was provided which could be consulted by $S$ at any time. Fach $S$ learned one list under each of the response availability conditions. The order of response availability conditions and assignment of lists to conditions were counterbalanced across Ss. During the paired-associate task, blank trials were not allowed; Ss were required to provide a response for every stimulus on every trial before the correct response was shown.

\section{RESULTS}

Preliminary analyses showed that the effects of the response-availability conditions and of the two lists were not significant, so data from these conditions were combined for additional analyses. The $\mathrm{Ss}$ found their second lists much easier to learn than their first lists $(F=14.69, \mathrm{df}=1 / 36$; $p<.001$ ) for an analysis of total errors. Data from Lists 1 and 2 are reported separately in the analyses which follow.

The Ss tended to guess on unlearned items with responses appropriate to other unlearned items. Chance levels for each S-item were obtained by summing the proportions of learned-item responses which would be guessed on each trial if $\mathrm{S}$ were to guess randomly from the entire response pool. These chance levels were consistently larger than the obtained number of guesses of responses to learned items $^{1} \quad(t=8.52$ and 3.41 , df $=19$ for Lists 1 and 2 , respectively; both $\mathrm{p}<.01$ ). On the other hand, the proportion of learned-item guesses was significantly greater than zero $(t=7.64$ and 11.87 , df $=19$ for Lists 1 and 2 , respectively; both $p<.01$ ). The results are illustrated in Fig. 1, which shows the proportion of learned items and the proportion of learned-response guesses during the four Vincent quartiles of the precriterion period. For both lists, the proportion of learned-item guesses was above zero, but well below the proportion of learned items, and increased with the

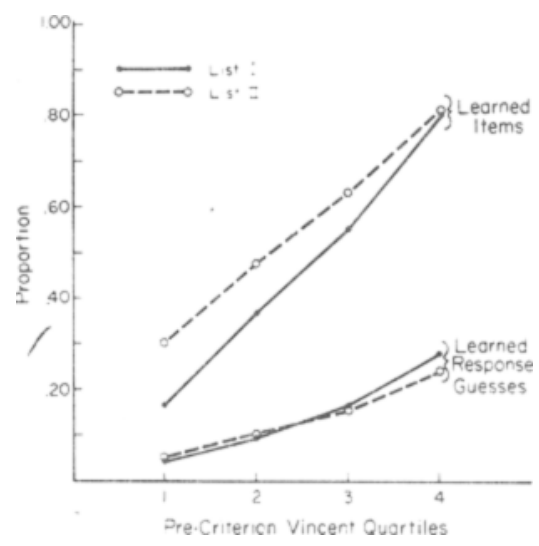

Fig. 1. Mean proportions of items learned and of learned responses used as guesses for unlearned items during the precriterion period. 


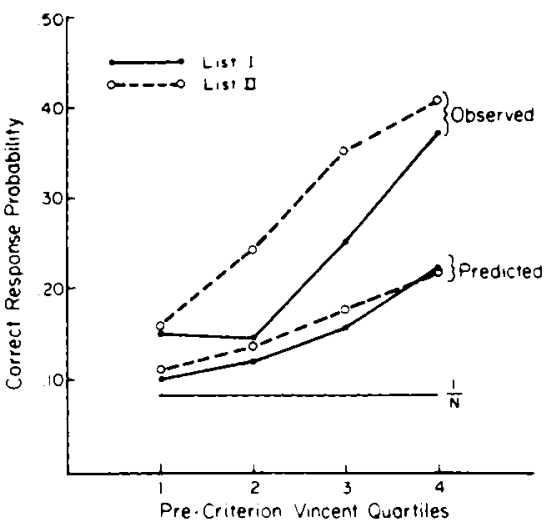

Fig. 2. Observed precriterion "stationarity" curves compared with those predicted from guessing-pool shrinkage alone.

proportion of learned items. The Ss tended to keep learned responses from the guessing pool for other items, but were less than perfect at doing so.

Figure 2 displays precriterion "stationarity" curves for the two lists, along with stationarity curves predicted from the pool-shrinkage assumption alone. For the predicted curve, each point represents the reciprocal of the mean number of items (across Ss) not yet learned during a precriterion quartile. As is obvious from Fig. 2, the pool-shrinkage notion underestimates the actual probability of a correct response by a considerable amount. [The nonstationarity exhibited in the observed data is highly significant $\left(x^{2}=75.50\right.$ and $31.67, \mathrm{df}=3$ for Lists 1 and $2 ; p<.01$ ). ]

Figure 3 displays related data, the probability of a correct response during the precriterion period as a function of the number of stimulus-response pairs reaching their trial of last error. For example, on any trial where exactly two pairs had reached the trial of last error, the probability of a correct response on the 10 unlearned pairs was calculated, pooled across all $\mathrm{Ss}$, and plotted at $\mathrm{N}-\mathrm{k}=10$. The theoretical probability at this point equals $1 / N-k=1 / 10$, on the basis of pool shrinkage from correct response elimination alone. As can be seen, Ss' performance was above this "chance" curve. Because there are different numbers of $S$-items at each point in Fig. 3, it was not possible to evaluate the significance of the difference using individual S's data. Instead, $t$ tests were computed considering each $\mathrm{N}-\mathrm{k}$ value as an experimental unit. These tests showed that the obtained probabilities were significantly higher than the theoretical "chance" probabilities $(t=4.14, \quad d f=10$; $p<01$ for List A and $t=2.49, \mathrm{df}=10$; $p<.05$ for List B). It should be noted that the decision to count any item as learned when it reached its trial of last error makes this test and these data conservative, since any item which was actually learned following its trial of last error would, if added to the observed data, make the probability of a correct response by guessing larger than indicated in Figs. 2 and 3.

The data presented thus far show that Ss do tend to eliminate from the guessing pool responses learned as correct to other stimulus terms but that their guessing efficiency is well above that due to this kind of pool shrinkage alone. While this could be due to factors such as incremental strengthening of the correct response, we prefer to seek an explanation in terms of other sources of pool shrinkage.

Restle (1964) has described several sources of difficulty in the learning of paired-associate materials, such as problems of stimulus discrimination and of response generalization. While these are undoubtedly sources of difficulty, they are also sources of increased guessing efficiency. For example, if S "knows" that a response is a number in the $80 \mathrm{~s}$ and there are only two such numbers in the response pool, his probability of being correct by chance is .50 , well above the $1 / 12$ possible by random guessing or any of the $1 / \mathrm{N}-\mathrm{k}$ values (except when $\mathrm{N}-\mathrm{k} \leqslant 2$ ). The same thing happens if he responds indiscriminately to Stimuli $B$ and $D$ with the response correct for one of them. Restle's sources of difficulty are sources of efficient guessing-pool reduction and thus are potential explanations for nonstationarity in precriterion performance curves.

Incorrect response data from the present study show clear evidence for the kinds of nonrandom guessing errors discussed by Restle (1964). 2 First, error totals showed that items learned with few errors provided few error responses for other items. These were primarily items with extreme responses (the highest and lowest numerical responses in each list) or items with distinctive responses (e.g., an item with response " 66 " in List A). The correlation between the number of errors for an item and the number of times its response was guessed to other items was .88 for List $A$ and .85 for List $\mathrm{B}$, both $\mathrm{p}<.01$.

Second, there was clear evidence of response generalization, both in terms of the absolute value of the response and in terms of common response elements. The commonest error response for a given pair was either one with a common element and/or

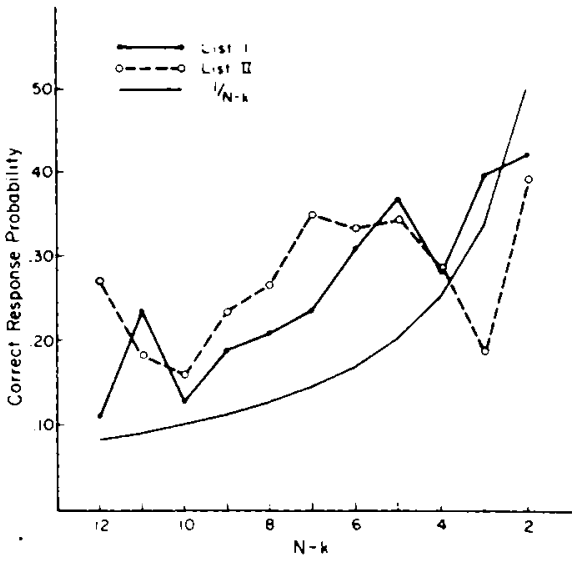

Fig. 3. Probability of a correct response during precriterion trials as a function of the estimated number of items not yet learned, $N-k$.

one numerically close for 18 of the 24 items, over both lists.

Third, there was some evidence for stimulus generalization, although the stimuli used here were too simple to allow this factor to have a very large effect. For example, the commonest error response to Item F-83 from List $B$ was the response to Item G-42; this is the clearest example, but for other items, guessing responses often came from stimuli which were alphabetically close. There was also a fairly high proportion of response guesses coming from acoustically similar stimuli.

\section{DISCUSSION}

Departures from stationarity in PA learning were shown to be partially due to increased guessing efficiency resulting from the elimination of learned responses from guessing pools for yet unlearned items. On precriterion trials, Ss avoided using responses from learned pairs and showed an increase in the probability of a correct guess on unlearned items. However, it was demonstrated that such pool shrinkage alone is insufficient to explain all the observed nonstationarity.

Further explanation of nonstationarity in terms of improved guessing efficiency was sought in the data by examining the different types of errors committed. It seems probable that a large part of the improved guessing resulted from the partial learning of $S-R$ pairs or, equivalently, the process of limiting the responses which could go with particular stimuli (Runquist, 1966).

The three sources of guessing-pool reduction that seem to be the major ones operating are: elimination of responses to easily learned items, elimination of responses due to partial learning of the correct response (one 
component of the response or its general value), and narrowing of the response pool due to stimulus confusion errors. As more and more items are learned on a list, the guessing pools for remaining unlearned items become smaller and smaller due to the operation of these factors, producing the nonstationary performance observed.

\section{REFERENCES}

BOWER, G. H. A model for response and training variables in paired-associate learning. Psychological Review, 1962,69, 34-53.

BREGMAN, A. S. Is recognition memory all-or-none? Journal of Verbal Learning \& Verbal Behavior, 1966, 5, 1-6.
MILLWARD, R. An all-or-none model for noncorrection routines with elimination of incorrect responses. Journal of Mathematical Psychology, 1964, 1 . 392-404.

NAHINSKY, I. D. Statistics and moments parameter estimates for a duoprocess paired-associate learning model. Journal of Mathematical Psychology, 1967, 4 , $140-150$

O'HARA, J. W., ERICKSON, J. R, Error elimination in paired-associate learning Journal of Verbal Learning \& Verbal Behavior, 1969, 8, 799-806.

RESTLE, F, Sources of difficulty in learning paired associates, In $R$. C. Atkinson (Ed.), Studies in mathematical psychology, Stanford, Calif: Stanford University Press, 1964.

RUNQUIST, W. N. Intralist interference as a function of list length and interstimulus similarity. Journal of Verbal Learning \& Verbal Behavior, $1966,5,7-13$.

SUPPES, P. \& GINSBERG, R. A fundamental property of all-or-none models: Binomial distribution responses prior to conditioning, with applications to concept formation in children. Psychological Review, 1963, 70, 139-161.

\section{NOTES}

1. An item was defined as learned following its trial of last error. This criterion is defensible since it is conservative with respect to the main conclusions, but undoubtedly overestimates the number of learned items on any given trial.

2. Complete confusion matrices are available from the authors: $98 \%$ of the errors were responses to other items on the list and the $2 \%$ extra-list errors occurred when the response pool was memorized.

\section{CURRENT LITERATURE ON VERBAL LEARNING}

CRAMER, P. (Williams College, Williamstown, Mass. 01267). Discrimination as a factor in semantic generalization. Journal of Experimental Psychology, 1971, 87, 396-400.

GEFFEN, G. (Monash University, Clayton, Victoria 3168, Australia), BRADSHAW, J. L., \& WALLACE, G. Interhemispheric effects on reaction time to verbal and nonverbal visual stimuli. Journal of Experimental Psychology, 1971, 87, 415-422.

KING, D. J. (State University College, Oswego, N.Y, 13126). Influence of interitem interval in the learning of connected discourse. Journal of Experimental Psychology, 1971, 87, 132-134

LIPPMAN, L. G. (Western Washington State College, Bellingham, Wash. 98225). Serial isolation effect as related to list-end demarcation. Journal of Experimental Psychology, 1971, 87, 135-137.

NELSON, D. L. (University of South Florida, Tampa, Fla. 33620), FOSSELMAN, J. R., \& PEEBLES J. W. Words as phonological sequences. Joumal of Experimental Psychology, 1971, 87, 361-366.

PETRICH, J. A. (University of
Pittsburgh, Pittsburgh, Pa. 15213). $R-S$ unlearning as a function of degree of S-R unlearning. Journal of Experimental Psychology, 1971, 87, 125-127.

ROBERGE, J. J. (Department of Educational Psychology, Temple University, Philadelphia, Pa. 19122). Journal of Experimental Psychology, 1971, 87, 127-129.

RUNQUIST, $W$. N. (University of Alberta, Edmonton, Alberta, Canada). Stimulus coding and interference in paired-associate learning. Journal of Experimental Psychology, 1971, 87, 373-377.

RUNQUIST, $\mathbf{W}$. $N$. (University of Alberta, Edmonton, Alberta, Canada), \& BLACKMORE, D. Individual differences in interference from stimulus similarity. Journal of Experimental Psychology, 1971, 87, 141-143.

SCHWARTZ, M. (University of Wisconsin, Madison, Wis. 53706). Subject-generated versus experimenter-supplied mediators in paired-associate learning. Journal of Experimental Psychology, 1971, 87, 389-395.

SCHWARTZ, M. (St. Francis Xavier University, Antigonish, Nova Scotia, Canada), \& BRYDEN, M. P. Coding factors in the learning of repeated digit sequences. Journal of Experimental Psychology, 1971, 87, 331-334.

TURNAGE, T. W. (University of Maryland, College Park, Md. 20742). Free recall of minimal serial lists. Journal of Experimental Psychology, 1971, 87, 378-382.

WALLACE, W. P. (University of Nevada, Reno, Nev. 89507), \& NAPPE, G. W. Re-pairing "rights" and "wrongs"' in verbal discrimination learning. Journal of Experimental Psychology, 1971, 87, $355-360$.

WEINSTOCK, R. B. (Mary Washington College of the University of Virginia), \& DALY, H. B. Response learning, association formation, and repeated testing effects in a paired-associate task. Journal of Experimental Psychology, 1971, 87, 343-347.

WILLIAMS, J. R. (Illinois State University, Normal, Пll. 61761), \& LEVIN, I. P., \& NORMAN, K. L. Mediation effects in verbal chaining paradigms as a function of associative strength and the number of stages. Journal of Experimental Psychology, 1971, 87, 348-354. 